\title{
ON LOBATCHEWSKY MANIFOLDS
}

\author{
BY SU-SHING CHEN $^{1}$
}

Communicated by S. S. Chern, September 22, 1973

Let $M$ be a complete, simply connected, $n$-dimensional Riemannian manifold with sectional curvature $K \leqq 0$. Eberlein in [7] and [9] has given the cone topology and a nice compactification $\bar{M}=M \cup M(\infty)$ of $M$. The boundary $M(\infty)$ of $M$ is the set of asymptotic classes of geodesics in $M . \bar{M}$ is homeomorphic to the closed unit ball in $\boldsymbol{R}^{n}$ and $M(\infty)$ is homeomorphic to $S^{n-1}$. Each isometry $\phi$ of $M$ extends to a homeomorphism of $\bar{M}$. Elements of the isometry group $I(M)$ can be classified according to their fixed points in $\bar{M}$. $\phi$ is called elliptic if $\phi$ has a fixed point in $M . \phi$ is called parabolic or axial if $\phi$ has exactly one fixed point or two fixed points in $M(\infty)$ respectively. If any two distinct points in the boundary $M(\infty)$ can be joined by a unique geodesic in $M$ (Axioms I and II), then $M$ is called a Lobatchewsky manifold for convenience. A complete, simply connected Riemannian manifold with sectional curvature $K \leqq c<0$ is a Lobatchewsky manifold.

In the sequel, we shall consider only Lobatchewsky manifolds $M$ and we shall assume that $I(M)$ acts effectively on $M$.

The main theorem is a description of complete homogeneous Riemannian manifolds with sectional curvature $K \leqq c<0$.

THEOREM 1. Let $M$ be a complete homogeneous Riemannian manifold with sectional curvature $K \leqq c<0$. Either $I(M)$ has a common fixed point in $M(\infty)$ or $M$ is a noncompact symmetric space of rank one.

The tool of this paper is the concept of the limit set of a subgroup $G$ of $I(M)$. The limit set $L(G)$ is the intersection with $M(\infty)$ of the closure of any orbit of $G$ in $M$. The limit set is independent of the choice of the orbit. If $A$ is a closed subset of $M(\infty)$ which contains more than one point and $A$ is invariant under a subgroup $G$ of $I(M)$, then $A \supset L(G)$. The totally geodesic hull $\langle A\rangle$ of a subset $A$ in $M(\infty)$ is the intersection of all totally geodesic submanifolds in $M$ whose boundaries contain $A$.

Let $G$ be a subgroup of $I(M)$. One obtains classification of $L(G)$ in the following manner: (1) $L(G)$ is empty, (2) $L(G)$ contains one point,

AMS (MOS) subject classifications (1970). Primary 53C30, $22 \mathrm{E} 40$.

1 The author is grateful to Professor L. Greenberg for his encouragement and guidance. 
(3) $L(G)$ contains two points, (4) $L(G)$ is an infinite, perfect and nowhere dense subset of $M(\infty),(5) L(G)=M(\infty)$. Consequently, one can obtain classification of subgroups of $I(M)$ according to their limit sets. A concrete classification of connected Lie subgroups of simple Lie groups of rank one has been accomplished in [5] and [6].

Here we present a unified version of the result in [5], [6] independent of Cartan's classification.

THEOREM 2. Let $M$ be a noncompact symmetric space of rank one. Let $G$ be a connected Lie subgroup of $I_{0}(M)$. Then one of the following holds:

(1) $G$ has a common fixed point in $M$;

(2) $G$ has a common fixed point in $M(\infty)$;

(3) G modulo a normal subgroup (isomorphic to a subgroup of $O(n-1)$ ) is the 1-parameter group of axial elements ${ }^{2}$ along the geodesic joining two fixed points;

(4) $G$ modulo a normal subgroup (isomorphic to a subgroup of $O(n-m)$, $m=\operatorname{dim}\langle L(G)\rangle)$ is the connected isometry group $I_{0}(S)$ of the totally geodesic submanifold $S=\langle L(G)\rangle$ which is a noncompact symmetric space of rank one;

(5) $G=I_{0}(M)$.

A consequence of Theorem 2 is the following

THEOREM 3. Let $M$ be a noncompact symmetric space of rank one and $G$ be a subgroup of $I_{0}(M)$. If there is no point in $\bar{M}$ and no proper totally geodesic submanifold in $M$ invariant under $G$, then $G$ is either discrete or dense in $I_{0}(M)$.

The above fact is related to Borel's density theorem [4] and Selberg's irreducible lattices [19].

We outline the proof of Theorems 1 and 2 by stating two main lemmas.

LEMMA 1. Let $M$ be a simply connected complete Riemannian manifold with $K \leqq c<0$ such that $I(M)$ acts effectively on $M$. Suppose that $G$ is a subgroup of $I(M)$ and $\langle L(G)\rangle=M$. If $L(G)$ contains more than two points, then the centralizer $Z(G, I(M))$ of $G$ in $I(M)$ is trivial. If, in addition, $G$ does not have a common fixed point in $M(\infty)$, then $G$ is semisimple.

LEMMA 2. Let $M$ be a noncompact symmetric space of rank one and $G$ be a Lie subgroup of $I_{0}(M)$ such that $M=\langle L(G)\rangle$. Suppose that $L(G)$ contains more than two points and $G$ does not have a common fixed point in $M(\infty)$. Then either $G$ is discrete or $G=I_{0}(M)$.

\footnotetext{
${ }^{2}$ The factored out normal subgroup of $G$ contains elliptic elements which leave the geodesic pointwise fixed but may rotate other points in $M$.
} 
Finally we state a theorem on the density of axial fixed points for Lobatchewsky manifolds. This fact is indispensable to geodesic and horospherical $G$-partition flows on a Lobatchewsky manifold. One can easily obtain a straightforward generalization of [10]. Furthermore one gets a corollary which generalizes a theorem [8] of Eberlein.

THEOREM 4. Let $M$ be a Lobatchewsky manifold and $G$ be a subgroup of $I(M)$. If $G$ contains axial elements and $G$ does not have a common fixed point in $M(\infty)$, then the fixed points of axial elements of $G$ are dense in $L(G) \times L(G)$.

COROLlARY. Let $M$ be a Lobatchewsky manifold and $G$ be a subgroup of $I(M)$. If $G$ does not have a common fixed point in $M(\infty)$ and $L(G)$ contains more than two points, then $G$ contains a free group with an infinite number of generators.

\section{REFERENCES}

1. D. V. Anosov, Geodesic flows on closed Riemannian manifolds of negative curvature, Trudy Mat. Inst. Steklov. 90 (1967) Proc. Steklov Inst. Math. 90 (1967). MR 36 \#7157; 39 \#3527.

2. V. I. Arnol'd and A. Avez, Problèmes ergodiques de la méchanique classique, Gauthier-Villars, Paris, 1967; English transl., Benjamin, New York, 1968. MR 35 \#334; 38 \#1233.

3. R. L. Bishop and B. O'Neill, Manifolds of negative curvature, Trans. Amer. Math. Soc. 145 (1969), 1-49. MR 40 \#4891.

4. A. Borel, Density properties for certain subgroups of semisimple groups without compact components, Ann. of Math. (2) 72 (1960), 179-188. MR 23 \#A964.

5. S. Chen and L. Greenberg, On hyperbolic spaces, Contributions to Analysis: A Collection of Papers Dedicated to Lipman Bers, Academic Press, New York (to appear).

6. S. Chen, On subgroups of the noncompact real exceptional Lie group $F_{4}$, Math. Ann. 204 (1973), 271-284.

7. P. Eberlein, Geodesic flows on negatively curved manifolds. I, Ann. of Math. 95 (1972), 492-510.

8. - Some properties of the fundamental group of a Fuchsian manifold, Invent. Math. 19 (1973), 5-13.

9. - Geodesic flow in certain manifolds without conjugate points, Trans. Amer. Math. Soc. 167 (1972), 151-170. MR 45 \#4453.

10. W. Gottschalk and G. Hedlund, Topological dynamics, Amer. Math. Soc. Colloq. Publ., vol. 36, Amer. Math. Soc., Providence, R.I., 1955. MR 17, 650.

11. L. Greenberg, Discrete subgroups of the Lorentz group, Math. Scand. 10 (1962), 85-107. MR 25 \#5128.

12. - Commensurable groups of moebius transformations, Conference on Discontinuous Groups and Riemann Surfaces, Ann. Math. Studies (to appear).

13. S. Helgason, Differential geometry and symmetric spaces, Pure and Appl. Math., vol. 12, Academic Press, New York, 1962. MR 26 \#2986.

14. W. Klingenberg, Geodätischer Fluß auf Mannigfaltigkeiten vom hyperbolischeu Typ, Invent. Math. 14 (1971), 63-82. MR 45 \#6034. 
15. S. Kobayashi and $\mathrm{K}$. Nomizu, Foundations of differential geometry. Vol. II, Interscience, New York, 1969.

16. H. B. Lawson, Jr. and S. T. Yau, Compact manifolds of nonpositive curvature, J. Differential Geometry 7 (1972), 211-228.

17. G. D. Mostow, Some new decomposition theorems for semisimple groups, Mem. Amer. Math. Soc. 14 (1955), 31-54. MR 16, 1087.

18. J. Nielsen, UUber Gruppen linearer Transformationen, Mitt. Math. Ges. Hamburg 8 (1940), part 2, 82-104. MR 2, 213.

19. A. Selberg, Recent developments in the theory of discontinuous groups of motions of symmetric spaces, Proc. Fifteenth Scand. Congress (Oslo, 1968), Lecture Notes in Math., vol. 118, Springer, Berlin, 1970, pp. 99-120. MR 41 \#8595.

20. C. L. Siegel, Bemerkung zu einem Staze von Jakob Nielsen, Mat. Tidsskr. B. 1950, 66-70. MR 12, 390.

Department of Mathematics, University of Florida, Gainesville, Florida 32601 